\title{
Obituary News September-2020
}

BMA would like to express deep condolence on deaths of the following notable physicians in recent past:

\begin{tabular}{|c|c|c|}
\hline S1.No. & Name \& Address & Date of Death \\
\hline 1 & $\begin{array}{l}\text { Dr. Main Uddin Ahmed } \\
\text { Assistant Professor of Medicine } \\
\text { Sylhet MAG Osmani Medical College }\end{array}$ & $15 / 4 / 2020$ \\
\hline 2 & $\begin{array}{l}\text { Professor Dr. Col (Ex) Moniruzzaman } \\
\text { Hematology \& Laboratory Medicine Specialist } \\
\text { Anwer Khan Modern Medical College Hospital, Dhaka }\end{array}$ & $03 / 5 / 2020$ \\
\hline 3 & $\begin{array}{l}\text { Professor Dr. Anisur Rahman } \\
\text { Forensic Medicine Specialist } \\
\text { Northern International Medical College, Dhaka. }\end{array}$ & $11 / 05 / 2020$ \\
\hline 4 & $\begin{array}{l}\text { Freedom Fighter Major (Ex) Dr. Abul Mukarim Md. Mohasin Uddin } \\
\text { Radiologist, Ex. Student of Mymensingh Medical College (11th Bach) }\end{array}$ & $12 / 05 / 2020$ \\
\hline 5 & $\begin{array}{l}\text { Dr. Md. Azizur Rahman Raju } \\
\text { Ex. Student of Mymensingh Medical College (13th Bach) }\end{array}$ & $18 / 05 / 2020$ \\
\hline 6 & $\begin{array}{l}\text { Dr. MA Matin } \\
\text { Member BMA Moulvibazar Branch } \\
\text { Assistant Director Helth, DGHS, Dhaka }\end{array}$ & $22 / 05 / 2020$ \\
\hline 7 & $\begin{array}{l}\text { Dr. Kazi Dilruba } \\
\text { Ex. Student, Sylhet MAG Osmani Medical College }\end{array}$ & $22 / 05 / 2020$ \\
\hline 8 & $\begin{array}{l}\text { Dr. SM Jafar Hossain } \\
\text { Medical Officer, Chattogram Maa-O-Shishu Hospital }\end{array}$ & $25 / 05 / 2020$ \\
\hline 9 & $\begin{array}{l}\text { Dr. Amina Khan } \\
\text { Senior Consultant, Gynaecology (Retd.) } \\
\text { Life Member, BMA }\end{array}$ & $26 / 05 / 2020$ \\
\hline 10 & $\begin{array}{l}\text { Dr. Abdur Rahman } \\
\text { Anesthesia and ICU Specialist }\end{array}$ & $26 / 05 / 2020$ \\
\hline 11 & $\begin{array}{l}\text { Dr. Md. Mosarraf Hossain } \\
\text { Ex-Head, Department of Orthopaedic } \\
\text { Sher E Bangla Medical College, Barihal }\end{array}$ & $27 / 05 / 2020$ \\
\hline 12 & $\begin{array}{l}\text { Dr. A F M Saidul Islam } \\
\text { Retd. Captain, Army Medical Core } \\
\text { Ex-Student, Sher E Bangla Medical college (1st Bach) }\end{array}$ & $28 / 05 / 2020$ \\
\hline 13 & $\begin{array}{l}\text { Dr. Wahiduzzaman Akand Bablu } \\
\text { Ex. Student, Sylhet MAG Osmani Medical College (14th Bach) }\end{array}$ & $31 / 05 / 2020$ \\
\hline 14 & $\begin{array}{l}\text { Dr. Manzoor Rashid Chowdhury } \\
\text { Retd. Consultant of Urology } \\
\text { Dhaka Medical College Hospital, Dhaka } \\
\text { Ex. Student, Sylhet MAG Osmani Medical College( 14th Bach) }\end{array}$ & $02 / 06 / 2020$ \\
\hline
\end{tabular}




\begin{tabular}{|c|c|c|}
\hline Sl.No. & Name \& Address & Date of Death \\
\hline 15 & $\begin{array}{l}\text { Dr. A.S.M. Ehsanul Karim } \\
\text { Associat Professor and Head, Department of Medicine } \\
\text { Marine City Medical College Hospital, Chattogram }\end{array}$ & 03/06/2020 \\
\hline 16 & $\begin{array}{l}\text { Dr. Md. Mohiuddin } \\
\text { Professor, Department of Microbiology } \\
\text { Ibrahim Medical College, Dhaka } \\
\text { Ex. Student of Chattogram Medical College( 17th Bach) }\end{array}$ & 03/06/2020 \\
\hline 17 & $\begin{array}{l}\text { Dr. K.M. Wahidul Haquee } \\
\text { Ex. Student, Sylhet MAG Osmani Medical College (10 Bach) }\end{array}$ & 03/06/2020 \\
\hline 18 & $\begin{array}{l}\text { Professor Dr. Habibur Rahman } \\
\text { Forensic Medicine Specialist }\end{array}$ & $04 / 06 / 2020$ \\
\hline 19 & $\begin{array}{l}\text { Dr. Muhidul Hasan } \\
\text { EMO, Chattogram Medical College \& Hospital }\end{array}$ & $04 / 06 / 2020$ \\
\hline 20 & $\begin{array}{l}\text { Professor Dr. N. I. Khan } \\
\text { Ex. Professor and Head Department of Medicine } \\
\text { Dhaka Medical College }\end{array}$ & $04 / 06 / 2020$ \\
\hline 21 & $\begin{array}{l}\text { Professor Dr. A.S.M. Golam Kibria } \\
\text { Ex. Chairman, Department of Urology } \\
\text { Bangabandhu Sheikh Mujib Medical University } \\
\text { Ex. Student of Sylhet MAG Osmani Medical College (6th Bach) }\end{array}$ & $04 / 06 / 2020$ \\
\hline 22 & $\begin{array}{l}\text { Dr. Ehsanul Kabir Chowdhury } \\
\text { Retd. Upazila Health \& Family Planning Officer } \\
\text { Ex. Student of Rangpur Medical College( } 4 \text { th Bach) }\end{array}$ & $04 / 06 / 2020$ \\
\hline 23 & $\begin{array}{l}\text { Dr. Abul Kashem Khan } \\
\text { Retd. Senior Medical Officer, EPZ, Saver } \\
\text { Ex. Student of Sylhet MAG Osmani Medical College (9th Bach) }\end{array}$ & $06 / 06 / 2020$ \\
\hline 24 & $\begin{array}{l}\text { Dr. Mirza Nazim Uddin } \\
\text { Senior Consultant and Director, Medical Services } \\
\text { Square Hospital, Dhaka } \\
\text { Life Member, BMA }\end{array}$ & $07 / 06 / 2020$ \\
\hline 25 & $\begin{array}{l}\text { Dr. Rajia Sultana } \\
\text { Ex. Student of Mymensing Medical College (16th Bach) }\end{array}$ & 08/06/2020 \\
\hline 26 & $\begin{array}{l}\text { Dr. Sakhawat Hossain } \\
\text { Consultant (Anesthesia), Labaid Hospital, Dhaka } \\
\text { Ex. Student of Community Based Medical College, } \\
\text { Mymensingh (5th Bach) }\end{array}$ & 08/06/2020 \\
\hline 27 & $\begin{array}{l}\text { Dr. Anwar Hossain } \\
\text { Chairman, Rahat Anwar Hospital, Barisal } \\
\text { Ex. Student of Sher E Bangla Medical College, Barihal (14th Bach) }\end{array}$ & $09 / 06 / 2020$ \\
\hline
\end{tabular}




\begin{tabular}{|c|c|c|}
\hline Sl.No. & Name $\&$ Address & Date of Death \\
\hline 28 & $\begin{array}{l}\text { Professor Dr. Jalilur Rahman } \\
\text { ICU Incharge and Senior Consultant } \\
\text { Impulse Hospital, Dhaka }\end{array}$ & $09 / 06 / 2020$ \\
\hline 29 & $\begin{array}{l}\text { Dr. Tanzila Rahman } \\
\text { Head of Quality, Department of Maternity, Marystops Bangladesh } \\
\text { Ex. Student of Z H Sikder Women's Medical College \& } \\
\text { Hospital, Barihal (4th Bach) }\end{array}$ & $10 / 06 / 2020$ \\
\hline 30 & $\begin{array}{l}\text { Professor Dr. Gazi jahirul Hasan } \\
\text { Professor of Pediatric Surgery, BSMMU } \\
\text { Ex. Student of Mymenging Medical College (17th Bach) }\end{array}$ & $12 / 06 / 2020$ \\
\hline 31 & $\begin{array}{l}\text { Dr. Mahmud Monwar } \\
\text { Assistant Professor, Department of Cardiology } \\
\text { National Heart Institute Hospital }\end{array}$ & $12 / 06 / 2020$ \\
\hline 32 & $\begin{array}{l}\text { Dr. A K M Fazlul Haque } \\
\text { Associate Professor, Department of Opthalmology } \\
\text { Z H Sikder Women's Medical College \& Hospital, Dhaka }\end{array}$ & $12 / 06 / 2020$ \\
\hline 33 & $\begin{array}{l}\text { Dr. Arif Hasan } \\
\text { Ex. Student of Dhaka Medical College (K-49 Bach) }\end{array}$ & $12 / 06 / 2020$ \\
\hline 34 & $\begin{array}{l}\text { Dr. Md. Sazzad Hossain } \\
\text { Head, Department of ICU } \\
\text { BRB Hospital, Dhaka, Retd. Associate Professor, Anesthesia } \\
\text { Ex. Student of Chattogram Medical College (20th Bach) }\end{array}$ & $13 / 06 / 2020$ \\
\hline 35 & $\begin{array}{l}\text { Dr. Sadekur Rahman } \\
\text { Life Member, BMA } \\
\text { Ex. Student of Chattogram Medical College (17th Bach) }\end{array}$ & $14 / 06 / 2020$ \\
\hline 37 & $\begin{array}{l}\text { Dr.Tawfikun Nesha } \\
\text { Retd. Additional Chief Medical Officer, BCIC } \\
\text { Ex. Student of Mymensingh Medical College (7th Bach) } \\
\text { Dr. ANM Abdul Hai } \\
\text { Ex. Civil Surgeon, Cox's Bazaar } \\
\text { Ex. Student of Sylhet MAG Osmani Medical College (7th Bach) }\end{array}$ & $15 / 06 / 2020$ \\
\hline 38 & $\begin{array}{l}\text { Professor Dr. A K M Mujibur Rahman } \\
\text { Ex. Director, Shahid Suhrawardy Medical College Hospital } \\
\text { Ex. Student, Sir Salimullah Medical College (4th Bach) }\end{array}$ & $16 / 06 / 2020$ \\
\hline 39 & $\begin{array}{l}\text { Dr. Md. Ashrafuzzaman } \\
\text { Retd. Associate Professor, Burn Unit, Dhaka Medical College } \\
\text { Ex. Student of Rangpur Medical College ( 8th Bach) }\end{array}$ & $17 / 06 / 2020$ \\
\hline 40 & $\begin{array}{l}\text { Dr. Md. Shah Abdul Ahad } \\
\text { Ex. Director, M Abdur Rahim Medical College Hospital, Dinajpur }\end{array}$ & $17 / 06 / 2020$ \\
\hline
\end{tabular}




\begin{tabular}{|c|c|c|}
\hline S1.No. & Name \& Address & Date of Death \\
\hline 41 & $\begin{array}{l}\text { Dr. Md. Nurul Haque } \\
\text { Ex. Student, Chattogram Medical College (38th Bach) }\end{array}$ & $17 / 06 / 2020$ \\
\hline 42 & $\begin{array}{l}\text { Dr. Md. Rafiqul Hayder Liton } \\
\text { Ragistrar, Department of Endocrinology } \\
\text { Saver Enam Medical College \& Hospital } \\
\text { Ex. Student of Sylhet MAG Osmani Medical College (23th Bach) }\end{array}$ & $17 / 06 / 2020$ \\
\hline 43 & $\begin{array}{l}\text { Dr. Md. Emdadullah Khan } \\
\text { Senior Consultant Dermatology } \\
\text { Ex. Student Barisal General Hospital \& Sher E Bangla Medical College (12th Bach) }\end{array}$ & $19 / 06 / 2020$ \\
\hline 44 & $\begin{array}{l}\text { Dr. Md. Shafiq Ahmed } \\
\text { Ex. Student, Sir Salimullah Medical College (1st Bach) }\end{array}$ & $20 / 06 / 2020$ \\
\hline 45 & $\begin{array}{l}\text { Dr. Mujibur Rahman Ripon } \\
\text { Associate Professor, Deptment of Pediatric } \\
\text { Central Medical College, Cumilla }\end{array}$ & $20 / 06 / 2020$ \\
\hline 46 & $\begin{array}{l}\text { Dr. Bazlur Rahman } \\
\text { General Surgeon, Impulse Hospital, Dhaka }\end{array}$ & $20 / 06 / 2020$ \\
\hline 47 & $\begin{array}{l}\text { Dr. Sunil Kumar Sarker } \\
\text { Retd. Associate Professor, Cardiac Surgery, NICVD, Dhaka }\end{array}$ & $21 / 06 / 2020$ \\
\hline 48 & $\begin{array}{l}\text { Dr. Lalit Kumar Dutt } \\
\text { ENT Specialist } \\
\text { Ex. Student, Chattogram Medical College (2th Bach) }\end{array}$ & $21 / 06 / 2020$ \\
\hline 49 & $\begin{array}{l}\text { Freedom Fighter Dr. Md. Ali Ajgar } \\
\text { Retd. Physician Narayanganj General Hospital }\end{array}$ & $23 / 06 / 2020$ \\
\hline 50 & $\begin{array}{l}\text { Dr. Upendra Nath Pal } \\
\text { Ex. Upazila Health and Family Planning Officer } \\
\text { Fakirhat Upjazila, Bagerhat, }\end{array}$ & $23 / 06 / 2020$ \\
\hline 51 & $\begin{array}{l}\text { Dr. Md. Yunus Ali Khan } \\
\text { Elderly Physician \& Eye Specialist, Shajadpur, Sirajgang } \\
\text { Ex. Student of Rajshahi Medical College ( } 4 \text { th Bach) }\end{array}$ & $24 / 06 / 2020$ \\
\hline 52 & $\begin{array}{l}\text { Dr. Md. Samirul Islam Babu } \\
\text { Associate Professor, Orthopedic Surgery, Chattogram Medical College } \\
\text { Life Member, BMA } \\
\text { Ex. Student of Sher E Bangla Medical college (19th Bach) }\end{array}$ & $24 / 06 / 2020$ \\
\hline 53 & $\begin{array}{l}\text { Dr. S M Saiful Isalm } \\
\text { Director \& Diabetes Specialist, Al Manar Hospital, Dhaka. } \\
\text { Ex. Student of Dhaka Medical college ( K-17 Bach) }\end{array}$ & $24 / 06 / 2020$ \\
\hline 54 & $\begin{array}{l}\text { Dr. Firoza Banu Minu } \\
\text { Ex. Student, Rajshahi Medical College (14th Bach) }\end{array}$ & $25 / 06 / 2020$ \\
\hline
\end{tabular}




\begin{tabular}{|c|c|c|}
\hline Sl.No. & Name $\&$ Address & Date of Death \\
\hline 55 & $\begin{array}{l}\text { Dr. Mohammad Hossain } \\
\text { Retd. Medical Officer, Chattogram City Corporation } \\
\text { Life Member, BMA } \\
\text { Ex. Student of Chattogram Medical College (17th Bach) }\end{array}$ & $26 / 06 / 2020$ \\
\hline 56 & $\begin{array}{l}\text { Dr. Md. Asaduzzaman } \\
\text { Medical Officer, ENT Oncology } \\
\text { National Institute of Cancer Research \& Hospital (NICRH) } \\
\text { Ex. Student of Sylhet MAG Osmani Medical College (30th Bach) }\end{array}$ & $27 / 06 / 2020$ \\
\hline 57 & $\begin{array}{l}\text { Professor Dr. Gopal Shankar Day } \\
\text { Ex. Head, Department of Psychiatry } \\
\text { Ex. Student of Sylhet MAG Osmani Medical College (11th Bach) }\end{array}$ & $27 / 06 / 2020$ \\
\hline 58 & $\begin{array}{l}\text { Professor. Dr. Golam Sarwar } \\
\text { Ex. Head, Department of Pediatric } \\
\text { International Medical College, Gazipur } \\
\text { Ex. Student of Chattogram Medical College( 15th Bach) }\end{array}$ & $01 / 07 / 2020$ \\
\hline 59 & $\begin{array}{l}\text { Dr.Md. Ruhul Amin } \\
\text { Eye Specialist and Surgeon } \\
\text { Director, Al Raji Hospital Dhaka }\end{array}$ & $01 / 07 / 2020$ \\
\hline 60 & $\begin{array}{l}\text { Professor Dr. M A Ohab } \\
\text { Medicin Specialist \& Ex. Head of Department } \\
\text { Holy Family Red Crescent Medical College, Dhaka }\end{array}$ & $01 / 07 / 2020$ \\
\hline 61 & $\begin{array}{l}\text { Professor Dr. Muntakim Chwudhary } \\
\text { Ex. Head, Department of Physiology } \\
\text { Sir Salimullah Medical College } \\
\text { Life Member, BMA } \\
\text { Ex. Student of Dhaka Medical college (K-26 Bach) }\end{array}$ & $04 / 07 / 2020$ \\
\hline 62 & $\begin{array}{l}\text { Professor Dr. A K M Nurul Anwar } \\
\text { Ex. Director General, Directorate General of Health Services } \\
\text { Life Member, BMA }\end{array}$ & 05/07/2020 \\
\hline 63 & $\begin{array}{l}\text { Dr. Md Sazzad Hossain } \\
\text { Civil Surgeon, Feni } \\
\text { Ex. Student of Sylhet MAG Osmani Medical College (25th Bach) }\end{array}$ & $07 / 07 / 2020$ \\
\hline 64 & $\begin{array}{l}\text { Dr. Sultana Latifa Jaman Irin } \\
\text { Registrar, Gynecology and Obstetrics } \\
\text { Chattogram Maa O Shishu Medical College Hospital } \\
\text { Ex. Student of Cumilla Medical College( 14rd Bach) }\end{array}$ & $14 / 07 / 2020$ \\
\hline 65 & $\begin{array}{l}\text { Dr. S M Noor Uddin Al Baki Rumi } \\
\text { Assistant Professor of Surgery, Kushtia Medical College } \\
\text { Ex. Student of Chattogram Medical College( } 3 \text { rd Bach) }\end{array}$ & $17 / 07 / 2020$ \\
\hline
\end{tabular}




\begin{tabular}{|c|c|c|}
\hline S1.No. & Name \& Address & Date of Death \\
\hline 66 & $\begin{array}{l}\text { Dr. Abdul Hamid } \\
\text { Retd. Senior Medical Officer, Bangladesh Post Office } \\
\text { Ex. Student of Rajshahi Medical College (3rd Bach) }\end{array}$ & $17 / 07 / 2020$ \\
\hline 67 & $\begin{array}{l}\text { Dr. Koel Karim (Kuhu) } \\
\text { Indoor Medical Officer (Medicine) } \\
\text { Dhaka National Medical College and Hospital } \\
\text { Ex. Student of Mymensingh Medical College (22th Bach) }\end{array}$ & 07/07/2020 \\
\hline 68 & $\begin{array}{l}\text { Professor. Abul Hossain Khan Chowdhary } \\
\text { Heart Specialist, Ex. Director, NICVD, Dhaka } \\
\text { Life Member, BMA } \\
\text { Ex. Student of Dhaka Medical College (K-29 Bach) }\end{array}$ & $18 / 07 / 2020$ \\
\hline 69 & $\begin{array}{l}\text { Professor Dr. T I M Abdullah Al Faruk } \\
\text { Principal, Popular Medical College, Life Member BMA } \\
\text { Ex. President \& Secretary Society of Surgeons of Bangladesh } \\
\text { Ex. Student of Sher-e-Bangla Medical College, Barishal (1st Bach) }\end{array}$ & $28 / 07 / 2020$ \\
\hline 70 & $\begin{array}{l}\text { Dr. Md. Nazrul Islam Chowdhury Taslim } \\
\text { Associate Professor, Orthopaedic Surgery } \\
\text { Chattogram Medical College, Life Member, BMA } \\
\text { Ex. Student of Sylhet MAG Osmani Medical College (21th Bach) }\end{array}$ & 03/08/2020 \\
\hline 71 & $\begin{array}{l}\text { Dr. F B M Abdul Latif } \\
\text { Director, Homeopathic and Alternative Medicine } \\
\text { Directorate General of Health Education } \\
\text { Life Member BMA }\end{array}$ & $06 / 08 / 2020$ \\
\hline 72 & $\begin{array}{l}\text { Dr. Md Golam Mustafa } \\
\text { Precident, BMA Rajbari Branch } \\
\text { Ex. Student of Chattogram Medical College (6th Bach) }\end{array}$ & 08/08/2020 \\
\hline 73 & $\begin{array}{l}\text { Dr. Rezwanul Bari Shamim } \\
\text { Assistant Professor Ortho Surgery } \\
\text { Shahid Monsur Ali Medical College, Sirajganj } \\
\text { Life Member, BMA } \\
\text { Ex. Student of Rajshahi Medical College (30th Bach) }\end{array}$ & 09/08/2020 \\
\hline 74 & $\begin{array}{l}\text { Professor Dr. Md. Mostaq Hossain Ansari } \\
\text { Retd. Professor, Department of Community Medicine } \\
\text { Rajshai Medical College, Life Member, BMA } \\
\text { Ex. Student of Rajshahi Medical College (17th Bach) }\end{array}$ & $10 / 08 / 2020$ \\
\hline 75 & $\begin{array}{l}\text { Professor Dr. Md. Asadul Haque Khan } \\
\text { Ex. Head, Department of ENT } \\
\text { Sir Salimullah Medical College } \\
\text { Ex. Student of Rajshahi Medical College (5th Bach) }\end{array}$ & $11 / 08 / 2020$ \\
\hline
\end{tabular}




\begin{tabular}{|c|c|c|}
\hline S1.No. & Name $\&$ Address & Date of Death \\
\hline 76 & $\begin{array}{l}\text { Dr. Aftab Uddin Ahmed } \\
\text { Retd. Upazila Health and Family Planning Officer } \\
\text { Life Member, BMA } \\
\text { Ex. Student of Rajshahi Medical College (1 4th Bach) }\end{array}$ & $16 / 08 / 2020$ \\
\hline 77 & $\begin{array}{l}\text { Dr. Md Abdur Rahman } \\
\text { Ex. Director, Directorate of Health } \\
\text { Life Member, BMA } \\
\text { Ex. Student of Rajshahi Medical College (14th Bach) }\end{array}$ & $16 / 08 / 2020$ \\
\hline 78 & $\begin{array}{l}\text { Dr. Syed Akhter Hossain } \\
\text { Principal, MATS, Moulobibazar } \\
\text { Retd. Deputy Director, Directorate of Family Planning } \\
\text { Life Member, BMA } \\
\text { Ex. Student of Sylhet MAG Osmani Medical College (7th Bach) }\end{array}$ & $20 / 08 / 2020$ \\
\hline 79 & $\begin{array}{l}\text { Dr. A B M Siddiqul Islam } \\
\text { Retd. Assistant Director IPH } \\
\text { Life Member, BMA } \\
\text { Ex. Student of Mymensingh Medical College (10th Bach) }\end{array}$ & $20 / 08 / 2020$ \\
\hline 80 & $\begin{array}{l}\text { Dr. Abdullah Al Mahmud } \\
\text { Elderly Physician } \\
\text { Life Member, BMA \& EC Member, BMA Moulobi bazar } \\
\text { Ex. Student of Sylhet MAG Osmani Medical College }\end{array}$ & $28 / 08 / 2020$ \\
\hline 81 & $\begin{array}{l}\text { Freedom Fighter Dr.Md. Abdul Matin Patwari } \\
\text { Ex. Civil Surgeon, Cumilla } \\
\text { Life Member BMA } \\
\text { Ex. Student of Mymensingh Medical College (11th Bach) }\end{array}$ & $28 / 08 / 2020$ \\
\hline
\end{tabular}

May Allah bless the departed souls.

Our heartiest commiseration to the deceased's family, our prayers are with them during this difficult moment of their life. 